\title{
HI Clouds Detected Toward Virgo with the Arecibo Legacy Fast ALFA Survey
}

\author{
Brian R. Kent ${ }^{1}$ \\ ${ }^{1}$ Center for Radiophysics and Space Research, Space Sciences Building, Cornell University, \\ Ithaca, NY 14853, USA \\ email: bkent@astro.cornell.edu
}

\begin{abstract}
The Arecibo Legacy Fast ALFA survey is in the process of yielding a complete HI dataset of the Virgo Cluster and its environs (Giovanelli et al. 2007, Kent et al., in preparation). Assuming a distance to Virgo of $16.7 \mathrm{Mpc}$, the minimum detectable HI mass by ALFALFA is of order $2 \times 10^{7} \mathrm{M}_{\odot}$. A number of the HI detections appear to have interesting properties. Some appear associated with, but offset from, low surface brightness optical counterparts; others, at larger spatial offsets, may be tidally related to optical counterparts. Yet another class includes detections which are not identifiable with any optical counterparts. We present the ALFALFA results on these objects in the Virgo region, as well as followup aperture synthesis observations obtained with the VLA.
\end{abstract}

Keywords. galaxies:distances and redshifts, galaxies:evolution, galaxies: formation, radio lines: galaxies, galaxies:halos, individual:Virgo cluster, galaxies:clusters, galaxies:interactions

\section{Introduction}

Regions of high galaxian density in the local Universe afford the opportunity to explore a wealth of morphological diversity and physical processes. Observations of the local Universe provide a census of the low luminosity and low mass populations and their kinematic and photometric properties. Covering large areas of sky with blind surveys is extremely useful in giving a homogeneous dataset that can be compared and correlated with observations at multiple wavelengths.

The Virgo Cluster is the nearest rich cluster of galaxies, at a distance of $16.7 \mathrm{Mpc}$. The $\sim 1300$ cataloged member galaxies of Virgo are morphologically segregated, with early type galaxies inhabiting the cluster centers, dominated by ellipticals M87 and M49, and late-type spirals dominating the outer periphery. Dwarfs are the most abundant class in Virgo, with over 850 members. Binggeli, Sandage \& Tammann (1985) carried out the first high quality optical survey of the cluster, releasing a catalog (VCC) that has served as an important standard of reference for Virgo studies. Redshift measurements show that several subclumps in the outer periphery lie behind the main cluster centered around M87 (Binggeli, Tammann, \& Sandage 1987). As the cluster progresses toward a state of virial equilibrium, many galaxies in the outlying periphery of Virgo are moving at high speed while falling into the cluster (Binggeli, Popescu, \& Tammann 1993; Solanes et al. 2002). The cluster environment affects galaxies, evidenced by HI deficiency studies (Haynes \& Giovanelli 1986; Solanes et al. 2002), in the form of ram pressure interaction with the hot intracluster gas and other gravitational and hydrodynamic mechanisms. High resolution HI imaging has revealed disturbances and asymmetries in the gas disks, further evidence that the galaxies are interacting with the cluster environment (Cayatte 1990; Chung 2007). 
The $21 \mathrm{~cm}$ spectral line of neutral hydrogen is an extremely useful tool in determining the properties of galaxies. Redshifts, kinematics, and masses of gas content can all be obtained from global HI spectra. As such, large area blind HI surveys can provide invaluable statistics and information on the HI content of the local Universe, probing the faint end of the HI mass function within a cosmologically fair volume, revealing information about high mass galaxies, and tracing large scale structure. The Arecibo Legacy Fast ALFA (ALFALFA; Giovanelli et al. 2005a; Giovanelli et al. 2005b) extragalactic HI survey is specifically designed to provide a large homogeneous dataset of $\mathrm{HI}$ measurements for $c z_{\odot} \lesssim 18000 \mathrm{~km} \mathrm{~s}^{-1}$, able to detect as low as $M_{H I} \sim 2 \times 10^{7} M_{\odot}$ at the distance of Virgo. The blind survey will cover a total of $7000 \mathrm{deg}^{2}$ of high galactic latitude sky visible from Arecibo, including the full region of the Virgo cluster and its environs, as well as many other cluster and and groups in the local Universe. ALFALFA will serve as an important second generation HI survey to the successful HIPASS survey (Barnes et al. 2001), improving in sensitivity, bandwidth, and spectral resolution.

\section{ALFALFA Observations of the Virgo Cluster region}

The Virgo cluster and its surrounding periphery are a primary target for ALFALFA, as the cluster is easily accessible to the Arecibo telescope. An effort has been made to prioritize and complete coverage of that region by ALFALFA. The current Virgo catalogs encompass data taken in the Spring 2005 and 2006 observing sessions (Giovanelli et al. 2007 and Kent et al. in preparation). ALFALFA is conducted with the 7-element ALFA receiver in meridian transit drift mode. Spectra are sampled at a rate of $1 \mathrm{~Hz}$. A $100 \mathrm{MHz}$ bandwidth with 4096 channels yields a spectral resolution of $24.4 \mathrm{kHz}\left(5.1 \mathrm{~km} \mathrm{~s}^{-1}\right.$ at the $21 \mathrm{~cm}$ HI line). Drift scans are recorded in ten minute intervals, with a calibration noise diode signal inserted into the system between scans . After conversion into the IDL environment, software routines developed at Cornell are used for automated bandpass calibration and user-controlled radio frequency interference flagging and quality monitoring. Completed drifts are then combined into regularly gridded data cubes that encompass $2.4 \times 2.4$ degrees of sky. The data cubes are split into 1024 channel redshift sections overlapping by $\sim 1000 \mathrm{~km} \mathrm{~s}^{-1}$ in redshift space. Signal extraction is completed in Fourier space using an automated matched-filter algorithm (Saintonge 2007a) and candidate detections are confirmed by eye in a custom made visualization environment. Detections are corroborated with optical imaging from the Digital Sky Survey and SDSS; fluxes and $c z_{\odot}$ measurements are extracted from integrated spectral profiles. Published survey catalogs and spectra are available at http://arecibo.tc.cornell.edu/hiarchive/alfalfa/. In addition, National Virtual Observatory protocols allow catalog access via other websites through a cone search service. The whole data processing pipeline of ALFALFA is embedded in an environment that facilitates cross-referencing into other data sources.

The data presented in this paper encompass $\sim 240 \mathrm{deg}^{2}$ of sky from $11^{h} 44^{m}<$ R.A. $(\mathrm{J} 2000)<14^{h} 00^{m}$ and $08^{\circ} 00^{\prime}<$ Dec. $(\mathrm{J} 2000)<16^{\circ} 00^{\prime}$. Over 1300 sources were detected out to $c z_{\odot} \sim 18,000 \mathrm{~km} \mathrm{~s}^{-1}$. The sky distribution of galaxies for $c z_{\odot}<3000 \mathrm{~km} \mathrm{~s}^{-1}$ around the Virgo core region is shown in Figure 1. In this region, ALFALFA detects 5.5 sources $\mathrm{deg}^{-2}$. A velocity distribution of the entire catalog is depicted in Figure 2. The dark histogram in the same figure shows the galaxies detected for the same area of sky by the HIPASS Survey (Wong et al. 2006). The overdensity of sources near $c z_{\odot} \sim 1000 \mathrm{~km} \mathrm{~s}^{-1}$ indicates the Virgo cluster population. The histogram clearly shows large scale structure beyond Virgo, most prominently with the Coma supercluster regime at $c z_{\odot} \sim 7000 \mathrm{~km} \mathrm{~s}^{-1}$. Statistics of this dataset are shown in Figure 3, displaying the signal-to-noise and flux integral versus velocity width of the HI sources. The signal-to-noise limit of 6.5 is shown 


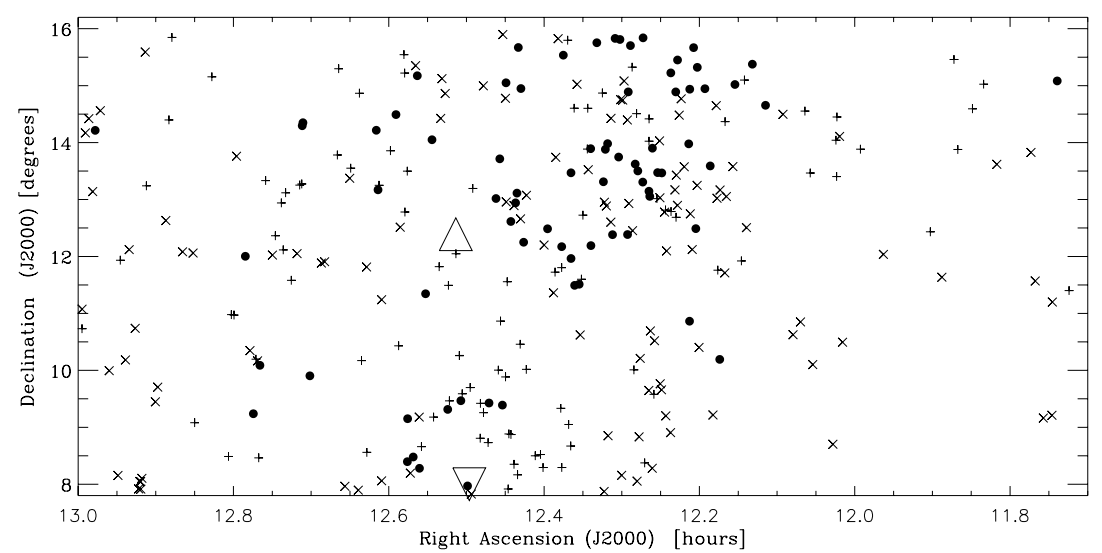

Figure 1. Sky distribution of galaxies for $c z_{\odot}<3000 \mathrm{~km} \mathrm{~s}^{-1}$ in the Virgo region so far detected by the ALFALFA survey. Large cluster galaxies M87 and M49 are indicated by the upward and downward pointing open triangles respectively. Filled circles indicate galaxies with $c z_{\odot}<500 \mathrm{~km} \mathrm{~s}^{-1}$, plus signs $(+)$ indicate galaxies with $500<c z_{\odot}<1500 \mathrm{~km} \mathrm{~s}^{-1}$, and $\times$ symbols indicate galaxies with $c z_{\odot}>1500 \mathrm{~km} \mathrm{~s}^{-1}$.

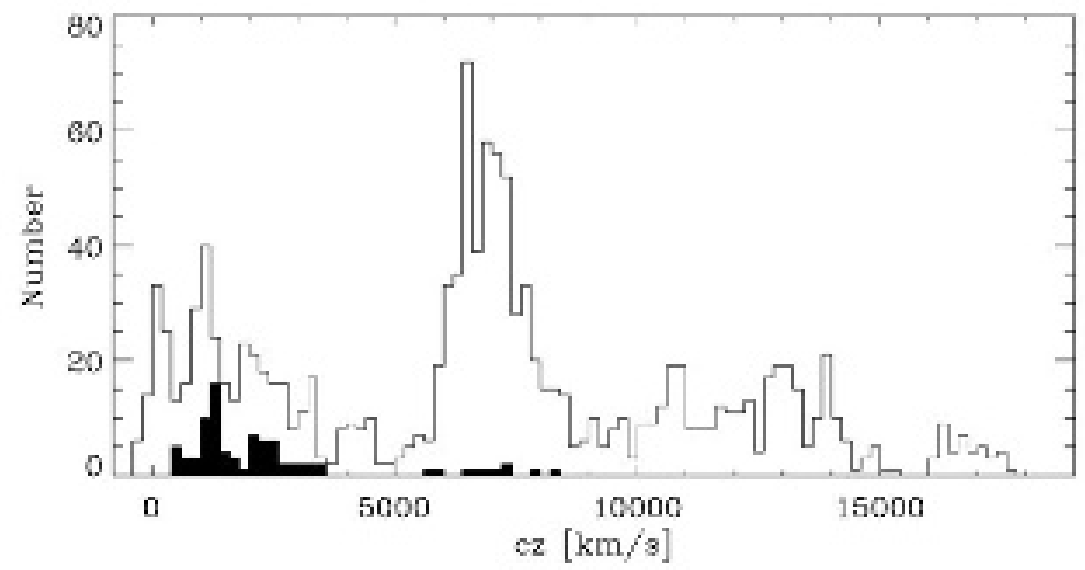

Figure 2. $c z_{\odot}$ distribution of detections for all galaxies in the catalogs of Giovanelli et al. (2007) and Kent et al. (in preparation). The dark histogram shows the distribution of HIPASS sources for the same region (Wong et al. 2006). Notable features showing large scale structure include the Virgo cluster population at $c z_{\odot} \sim 1000 \mathrm{~km} \mathrm{~s}^{-1}$ and the Coma supercluster regime at $c z_{\odot} \sim 7000 \mathrm{~km} \mathrm{~s}^{-1}$.

in the bottom plot as a function of velocity width; it is expected to increase as the square root of the velocity width (dotted line) for widths $\lesssim 300 \mathrm{~km} \mathrm{~s}^{-1}$ and linearly for larger widths, as observed.

When compared with the remainder of the survey, the Virgo area component of ALFALFA will allow for the comparison between the HI mass function in the cluster and in the field. However, because of large peculiar velocities toward the Virgo region, primary distance indicators (TRGB, SBF) will be needed to determine an accurate cluster HI mass function. Figure 4 shows the detections' HI mass vs distance, determined by using the results of mass flow models (Tonry et al. 2000; Masters 2005), except for objects within the Virgo cluster region, which are all placed at a distance of $16.7 \mathrm{Mpc}$. 

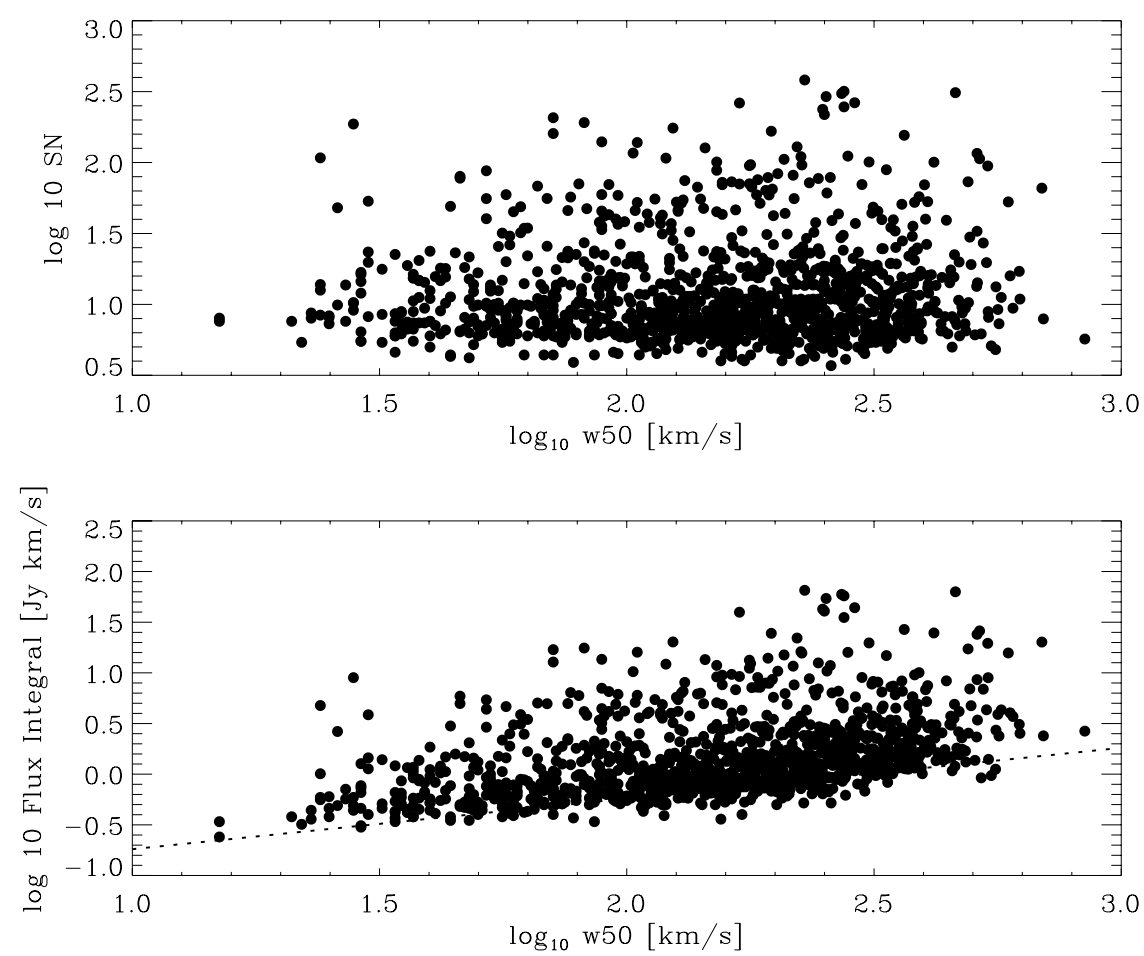

Figure 3. The top figure shows the $\mathrm{S} / \mathrm{N}$ vs. velocity width for the Virgo region sources detected with ALFALFA. The bottom figure shows the integrated flux vs. velocity width, with the dotted line indicating a $\mathrm{S} / \mathrm{N}$ limit of 6.5 .

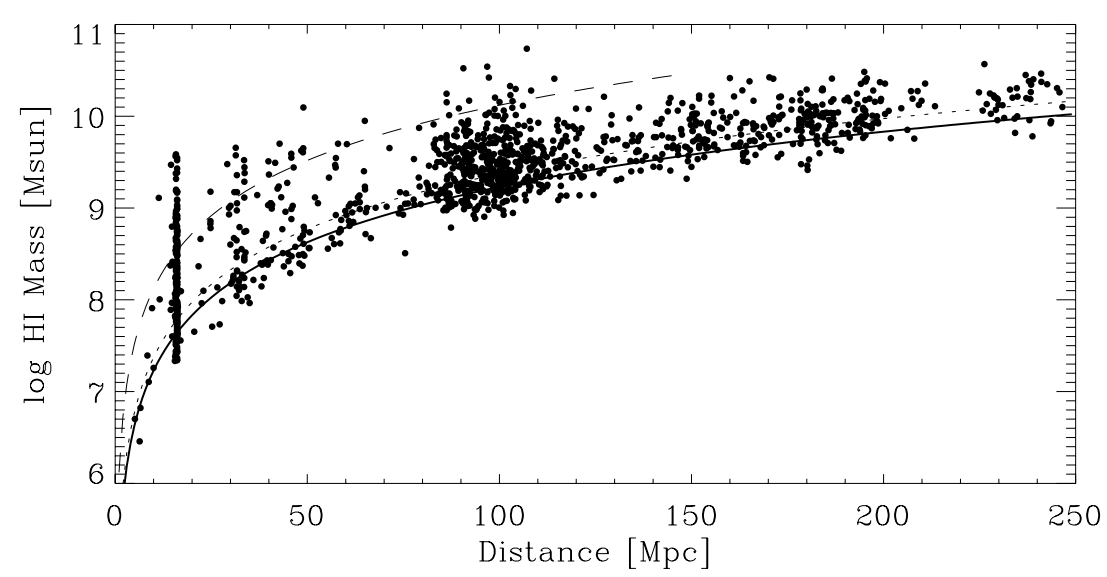

Figure 4. HI mass vs. distance for the Virgo region sources presented in this paper. Assumed Virgo cluster members are seen as a vertical line near $16.7 \mathrm{Mpc}$. The top dashed line indicates a flux integral of $5.6 \mathrm{Jy} \mathrm{km} \mathrm{s}^{-1}$, corresponding to a HIPASS $5 \sigma$ detection. The middle dotted line indicates a flux integral of $1.0 \mathrm{Jy} \mathrm{km} \mathrm{s}^{-1}$, and the lowest solid line indicates 0.72 Jy $\mathrm{km} \mathrm{s}^{-1}$ limit for the ALFALFA survey. All three lines are computed with a source velocity width $W_{\mathrm{FWHM}}=200 \mathrm{~km} \mathrm{~s}^{-1}$. 
Table 1. ALFALFA: Optically Unseen Detections Toward Virgo

\begin{tabular}{|c|c|c|c|c|c|c|c|c|}
\hline Cloud ID & $\alpha$ & $\delta$ & $c z_{\odot}$ & $W_{\text {FWHM }}$ & $F_{c}$ & $\mathrm{~S} / \mathrm{N}$ & $\log _{10} M_{H I}$ & $\mathrm{~d}_{M 87}$ \\
\hline Units & $\mathrm{J} 2000$ & J2000 & $\left(\mathrm{km} \mathrm{s}^{-1}\right)$ & $\left(\mathrm{km} \mathrm{s}^{-1}\right)$ & $\left(\mathrm{Jy} \mathrm{km} \mathrm{s}^{-1}\right)$ & - & $M_{\odot}$ & $\operatorname{deg}$ \\
\hline $1^{\mathrm{a}}$ & 120244.4 & +140456 & $1121 \pm 1$ & $22 \pm 2$ & $0.30 \pm 0.02$ & 5.1 & 7.29 & 7.0 \\
\hline $2^{\mathrm{b}}$ & 120845.5 & +115517 & $1230 \pm 1$ & $29 \pm 2$ & $0.77 \pm 0.04$ & 11.6 & 7.63 & 5.4 \\
\hline $3^{\mathrm{b}}$ & 121341.8 & +125351 & $2235 \pm 2$ & $53 \pm 3$ & $1.21 \pm 0.07$ & 9.2 & 8.54 & 4.2 \\
\hline $4^{\mathrm{c}}$ & 122619.4 & +125330 & $2246 \pm 5$ & $135 \pm 11$ & $2.05 \pm 0.07$ & 14.4 & 8.77 & 1.2 \\
\hline $5^{\mathrm{d}}$ & 122954.7 & +075812 & $473 \pm 5$ & $30 \pm 10$ & $1.13 \pm 0.06$ & 10.9 & 7.87 & 4.4 \\
\hline $6 a^{\mathrm{e}}$ & 121755.5 & +144445 & $1984 \pm 1$ & $128 \pm 2$ & $2.09 \pm 0.06$ & 16.2 & 8.70 & 3.9 \\
\hline $6 b^{\mathrm{e}}$ & 121749.1 & +150452 & $2200 \pm 6$ & $40 \pm 13$ & $0.52 \pm 0.05$ & 5.0 & 8.16 & 4.1 \\
\hline $6 c^{\mathrm{e}}$ & 121733.8 & +142347 & $2111 \pm 10$ & $65 \pm 20$ & $0.57 \pm 0.04$ & 7.3 & 8.17 & 3.8 \\
\hline $7 a^{\mathrm{b}}$ & 122942.8 & +094154 & $524 \pm 7$ & $116 \pm 15$ & $1.16 \pm 0.07$ & 8.6 & 7.87 & 2.7 \\
\hline $7 b^{\mathrm{b}}$ & 123019.4 & +093518 & $603 \pm 4$ & $252 \pm 7$ & $2.56 \pm 0.09$ & 13.1 & 8.22 & 2.8 \\
\hline $7 c^{\mathrm{b}}$ & 123025.8 & +092801 & $488 \pm 5$ & $62 \pm 11$ & $2.48 \pm 0.07$ & 21.2 & 8.21 & 2.9 \\
\hline $7 d^{\mathrm{b}}$ & 123119.0 & +092749 & $607 \pm 4$ & $56 \pm 7$ & $0.72 \pm 0.06$ & 6.5 & 7.67 & 2.9 \\
\hline $7 e^{\mathrm{b}}$ & 123126.7 & +091852 & $480 \pm 10$ & $53 \pm 21$ & $0.91 \pm 0.06$ & 7.6 & 7.77 & 3.1 \\
\hline $8 a^{\mathrm{f}}$ & 125504.3 & +080613 & $2629 \pm 3$ & $71 \pm 7$ & $0.72 \pm 0.07$ & 6.4 & 8.43 & 7.3 \\
\hline $8 b^{\mathrm{f}}$ & 125510.2 & +080244 & $2754 \pm 14$ & $407 \pm 27$ & $2.91 \pm 0.12$ & 9.4 & 9.08 & 7.4 \\
\hline $8 c^{\mathrm{f}}$ & 125513.7 & +080251 & $2771 \pm 4$ & $292 \pm 7$ & $2.52 \pm 0.10$ & 10.9 & 9.02 & 7.4 \\
\hline
\end{tabular}

a) Confirmed by follow-up, high sensitivity observation at Arecibo.

b) VLA maps obtained, processing underway.

c) VIRGOHI 4, Davies et al. (2004), VLA map by Oosterloo et al. (2005).

d) Vicinity of M49, Sancisi et al. (1987); synthesis data by Henning et al. (1993).

e) Clumps in VIRGOHI 21, WSRT data by Minchin et al. (2005).

f) NGC 4795/4796 group.

\section{ALFALFA HI Clouds in Virgo}

A small subgroup of objects have been found to have no apparent optical counterparts in the first Virgo portion of the ALFALFA survey (Kent et al. 2007). Here we report on eight detections, several with multiple components. With the exception of one, all are within the canonical sky and $c z_{\odot}$ bounds of the Virgo Cluster periphery defined in Binggeli, Popescu, \& Tammann (1993). Other objects of interest are discussed in the presentations of Giovanelli and Koopmann in these proceedings. The HI measurements and data of these clouds are listed in Table 1. The Table 1 columns are described as follows:

Col.(1) - Cloud ID number

Col.(2 \& 3) - HI source center coordinates (J2000); these positions are typically accurate to within $24^{\prime \prime}$ or better (see Giovanelli et al. 2007)

Col.(4) - Heliocentric velocity in $\mathrm{km} \mathrm{s}^{-1}$

Col.(5) - Velocity width measured at half peak power in $\mathrm{km} \mathrm{s}^{-1}$

Col.(6) - Integrated flux in Jy $\mathrm{km} \mathrm{s}^{-1}$

Col.(7) - Signal to noise ratio

Col.(8) - Base 10 logarithm of the HI mass in solar units, assuming HI is optically thin Col.(9) - Angular distance from M87 in degrees

Figure 5 shows these HI detections overlaid on a smoothed ROSAT X-ray map (Snowden et al. 1995). These optically "dark" objects are not detected in large numbers 


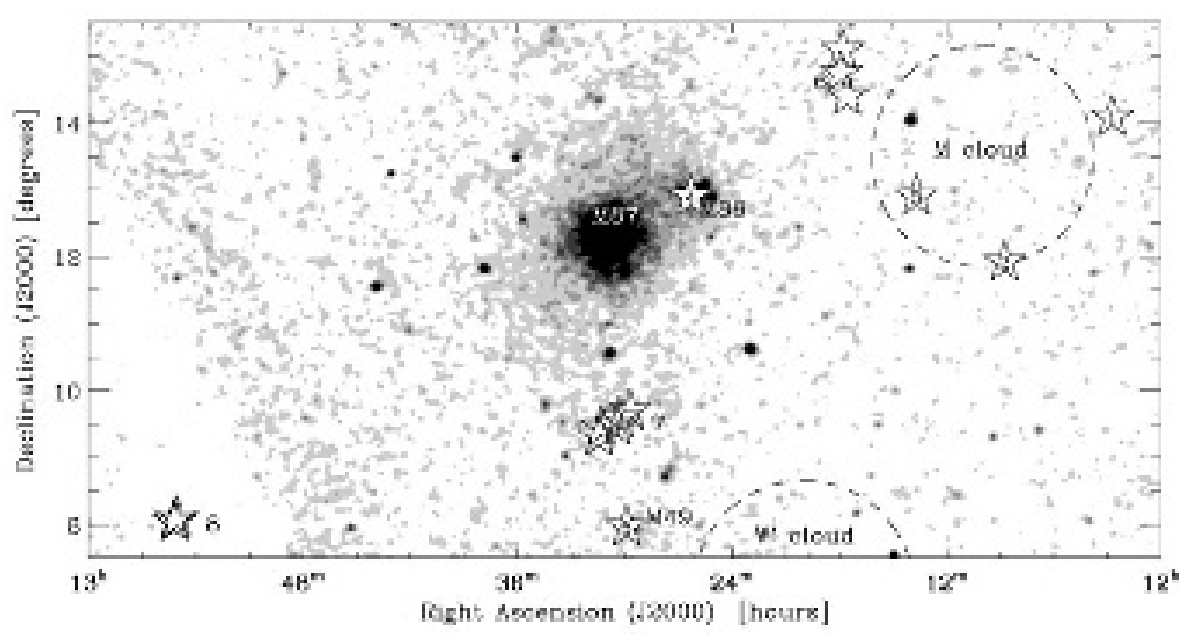

Figure 5. Optically unseen HI detections presented in this paper. The ROSAT X-ray background has been smoothed with a $5^{\prime}$ Gaussian kernel. The $\mathrm{M}$ and $\mathrm{W}^{\prime}$ clouds are outlined by dashed circles (Binggeli, Popescu, \& Tammann (1993). The numbering corresponds to the cloud identifiers given in Column 1 of Table 1.

toward Virgo, and no detections sharing similar characteristics have been found toward the ALFALFA anti-Virgo region (Saintonge et al. 2007b). In the high galaxian density environment of Virgo and its periphery, ram pressure stripping and high speed interaction are likely to take place, and gas removal from disks is more frequent.

Galaxy harassment also has been put forth as a strong influence on cluster evolution; galaxies can be stripped of their gas during high speed encounters with other cluster member and the cluster's potential (Moore et al. 1996). Many large disk galaxies inhabiting the outer parts of Virgo show evidence of having undergone some sort of dynamical interaction. HI studies of selected galaxies have shown that the HI disks are disturbed and/or asymmetric.

Previous studies have shown examples where the fingerprint of cluster interaction can clearly be seen. An HI plume extending from NGC $4388\left(c z_{\odot}=2524 \mathrm{~km} \mathrm{~s}^{-1}\right.$; Davies et al. 2004; Oosterloo \& van Gorkom 2005) was also detected in the ALFALFA survey (Table 1, Cloud 4). This feature has been attributed to interaction between the galaxy and the hot intracluster gas. Another example is listed as Cloud 5, likely a tidal interaction between dwarf irregular UGC 7636 and M49, the large galaxy central to the Virgo B subcluster.

Another object of note is Cloud 6. Originally detected with the Jodrell Bank telescope(Davies et al. 2004; Minchin et al. 2005a) and dubbed VIRGOHI 21, this detection has been tidally linked to the nearby large spiral NGC 4254. Galaxy harassment likely plays a role in the formation of such a tail, which extends over $250 \mathrm{kpc}$ north of the associated spiral (Haynes, Giovanelli \& Kent 2007). A more detailed description can be found in these proceedings by Giovanelli. Also presented are the results of modeling the interaction that my have caused it in Duc (these proceedings) and Duc \& Bournaud (2007).

Cloud 8 is discussed here although the associated SB0/a galaxy NGC $4795\left(c z_{\odot}=2781\right.$ $\left.\mathrm{km} \mathrm{s}^{-1}\right)$ and dwarf NGC $4796\left(c z_{\odot}=2406 \mathrm{~km} \mathrm{~s}^{-1}\right)$ lie behind Virgo. The presence of anomalous $\mathrm{HI}$ in this system was noted by Hoffman et al. (1989) and Duprie \& Schneider 


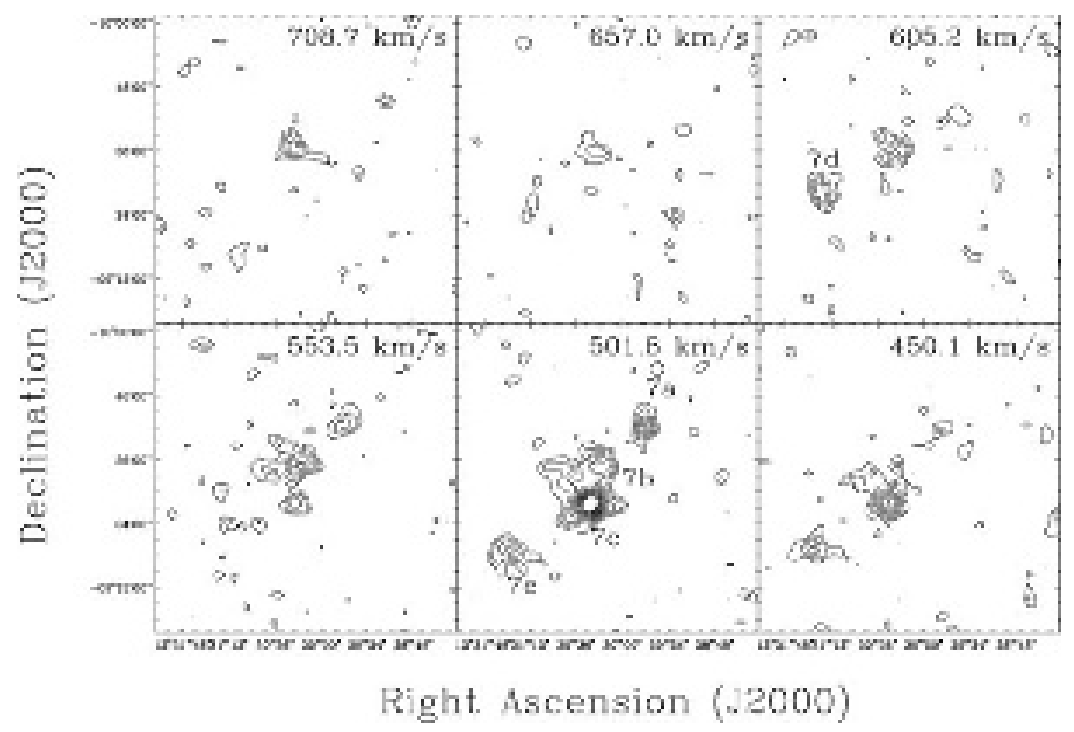

Figure 6. ALFALFA moment zero HI maps for Cloud 7 listed in Table 1. Each map is integrated over $50 \mathrm{~km} \mathrm{~s}^{-1}$ centered at the $c z_{\odot}$ value indicated in the upper right corners. Map contours are for 120, 200, 300, 400, 500, 600, 700, 800, 900, and $1000 \mathrm{mJy}$ beam $^{-1} \mathrm{~km} \mathrm{~s}^{-1}$ flux levels.

(1996). The ALFALFA map shows the HI is in clouds lying outside the optical disks of these galaxies.

The remaining objects in Table 1 (Clouds $1,2,3, \& 7$ ) are isolated, in that they are not obviously connected with large galaxies in their respective vicinities, nor can they be seen to be associated with faint but visible optical counterparts. Cloud group 7 is a HI complex detected during the Spring 2005 observing runs. The complex is composed of five components, stretched over $200 \mathrm{kpc}$ at the Virgo distance, situated between M87 and M49 on the sky. Moment zero maps of the complex showing the components are shown in Figure 6. Each map is integrated over $50 \mathrm{~km} / \mathrm{s}$, centered at the velocities shown in the upper right corners. Maps show flux contours at the 120, 200, 300, 400, 500, 600, $700,800,900$, and 1000 mJy beam ${ }^{-1} \mathrm{~km} \mathrm{~s}^{-1}$ flux levels. The velocity dispersion of the system is $\sim 250 \mathrm{~km} \mathrm{~s}^{-1}$ in extent. Aperture synthesis observations of this system have been obtained with the VLA and are in preparation for publication (Kent et al. 2007).

Clouds 1, 2, \& 3 share similar characteristics to each other, in that they are narrow sources $\left(W_{\mathrm{FWHM}} \lesssim 50 \mathrm{~km} \mathrm{~s}^{-1}\right)$, unresolved by the Arecibo beam, and all located in the western periphery of Virgo. At the Virgo distance, these objects are of order $10 \mathrm{kpc}$ or less in diameter. This region is also home to a population making up the so-called " $\mathrm{M}$ " Cloud (Binggeli, Sandage, \& Tammann 1993). This subgroup is thought to lie behind the main A cluster around M87; the velocity of the "M" Cloud population with respect to the systemic Virgo velocity also suggests that many of these galaxies are currently falling into the main cluster. These objects are far removed from the cluster core, and it is unlikely that hydrodynamical processes are the main instigator of this detachment from spiral disks. Instead, gravitational interaction with the cluster itself or high speed encounters are more likely to produce such debris. Such action within the cluster periphery can yield higher probabilities of tidal debris being found in this region. However, it remains a possibility that these objects could be associated with very small lower surface brightness dwarf galaxies or be veritable "dark galaxies". Further optical followup will shed light on the issue. 


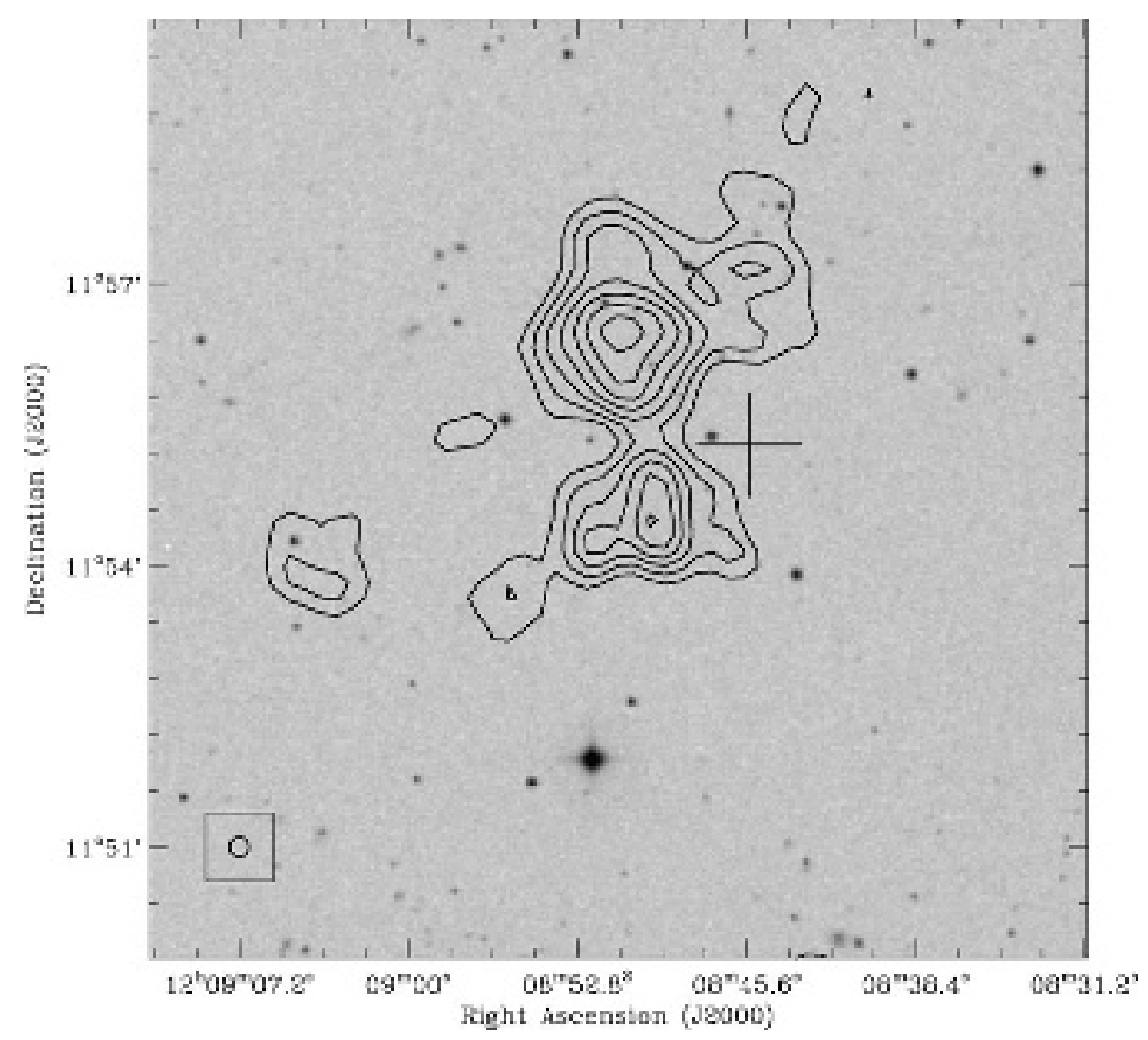

Figure 7. VLA C-array moment zero HI map of Cloud 2 from Table 1. The map was created using uniform weighting. The map rms is $\sigma=3.8 \mathrm{mJy}_{\text {beam }}{ }^{-1} \mathrm{~km} \mathrm{~s}^{-1}$, with contours indicating $3,4,5,6,7,8$ and $9 \sigma$. The large cross indicates the centroid of the Arecibo detection $\left(3.8^{\prime} \times\right.$ $3.3^{\prime}$ beam). The synthesized beam size is indicated in the lower left corner.

Analysis of aperture synthesis observations obtained with the VLA has shown that Cloud 2 can be resolved. The experiment utilized 12 hours of observation in C-configuration, centered on the Arecibo centroid for Cloud 2 given in Table 1. The backend setup consisted of a $1.56 \mathrm{MHz}$ bandwidth centered at $c z_{\odot}=1230 \mathrm{~km} \mathrm{~s}^{-1}$, with 128 channels, yielding a spectral resolution of $12.2 \mathrm{kHz}\left(2.5 \mathrm{~km} \mathrm{~s}^{-1}\right.$ at the $\left.21 \mathrm{~cm} \mathrm{HI} \mathrm{line}\right)$. A data cube using uniform weighting was created, with pixel elements smoothed to $10^{\prime \prime}$. A preliminary moment map was made from this cube, and is shown in Figure 7 . The map rms is $\sigma=3.8$ mJy beam $^{-1} \mathrm{~km} \mathrm{~s}^{-1}$, with contours indicating $3,4,5,6,7,8$ and $9 \sigma$. The large cross indicates the centroid of the Arecibo detection $\left(3.8^{\prime} \times 3.3^{\prime}\right.$ beam). The source is resolved into three extended clumps. The two main clumps are of high $\mathrm{S} / \mathrm{N}$, located near the pointing center and appear to be connected by a bridge. The peaks are separated by $\sim 2^{\prime}$ in space ( $\sim 9 \mathrm{kpc}$ at the Virgo distance). A third detection lies $\sim 3^{\prime}$ southeast of the northern most clump. The small, compact group of HI detections composing Cloud 2 are spread over no more than $15^{\prime}$ to $20^{\prime}$ in extent, with a velocity spread of $\sim 20 \mathrm{~km} \mathrm{~s}^{-1}$. The aperture synthesis data reveals that Cloud 2 is similar to the Cloud 7 complex, albeit on different size scales. Aperture synthesis observations have also been obtained for other cloud complexes in Table 1 and will be presented in a later publication (Kent et al. 2007). 
Simple timing arguments can be employed to explore the dynamics of both these systems. The velocity differences between the components of Cloud 7 are $\sim 250 \mathrm{~km} \mathrm{~s}^{-1}$. Assuming that the global complex is not gravitationally bound, then the rate of separation between the cloud elements would be $\sim 250 \mathrm{kpc} \mathrm{Gyr}^{-1}$. A similar scenario is found with Cloud 2. A $20 \mathrm{~km} \mathrm{~s}^{-1}$ separation between the narrow, compact elements yields a separation rate of $\sim 20 \mathrm{kpc} \mathrm{Gyr}^{-1}$. In both cases, the clouds will disperse in a gigayear or less, well within a cluster crossing time. This is indicative that these objects could be transient in nature, which could indicate why they are not detected in large numbers.

\section{Summary}

An overview of the core Virgo cluster catalog and a listing of detections with no apparent optical counterparts have been presented. The catalog shows a vast improvement on the first generation of blind HI surveys with hundreds of new HI detections, greater sensitivity, and both spectral and spatial resolution. HI clouds that cannot be matched to optical counterparts are not detected in large numbers. Those that are detected lie in the Virgo periphery. ALFALFA and VLA maps reveal kinematics about some of the multi-component detections, suggesting that they can easily disperse within a cluster crossing time.

\section{Acknowledgements}

The Arecibo Observatory is part of the National Astronomy and Ionosphere Center which is operated by Cornell University under a cooperative agreement with the National Science Foundation.

The National Radio Astronomy Observatory is facility of the National Science Foundation operated under cooperative agreement by Associated Universities, Inc.

This research has made use of the NASA/IPAC Extragalactic Database (NED) which is operated by the Jet Propulsion Laboratory, California Institute of Technology, under contract with the National Aeronautics and Space Administration. This work has been supported by NSF grants AST-0307661, AST-0435697, and AST-0607007.

Funding for the SDSS and SDSS-II has been provided by the Alfred P. Sloan Foundation, the Participating Institutions, the National Science Foundation, the U.S. Department of Energy, the National Aeronautics and Space Administration, the Japanese Monbukagakusho, the Max Planck Society, and the Higher Education Council for England. The SDSS website is http://www.sdss.org/.

Skyview was developed and maintained under NASA ADP Grant NAS5-32068 under the auspices of the High Energy Astrophysics Science Archive Research Center at the Goddard Space Flight Center Laboratory of NASA.

This research has made use of data obtained from or software provided by the US National Virtual Observatory, which is sponsored by the National Science Foundation.

\section{References}

Barnes, D. G, Staveley-Smith, L., de Block, W. J. G., Oosterloo, T. Stewart, I. M. et al. 2001, MNRAS 322, 486

Binggeli, B., Sandage, A., \& Tammann, G. A. 1985, AJ 90, 1681

Binggeli, B., Tammann, G. A., \& Sandage, A. 1987, AJ 94, 251

Binggeli, B., Popescu, C. C., \& Tammann, G. A. 1993, A\&A 98, 275

Cayatte, V., van Gorkom, J. H., Balkowski, C., \& Kotanyi, C. 1990, AJ 100, 604

Chung, A., van Gorkom, J. H., Kenney, J. D. P., \& Vollmer, B. 2007, ApJ (Letters) 659, L115

Duc, P. -A., \& Bournaud, F. 2007, ApJ submitted 
Duprie, K., \& Schneider, S. E. 1996, AJ 112, 937

Giovanelli, R. et al. 2005a, $A J$ 130, 2598

Giovanelli, R. et al. 2005b, AJ 130, 2613

Giovanelli, R. et al.. 2007, AJ 133, 2569

Haynes, M. P., Giovanelli, R., \& Kent, B. R. 2007, ApJ (Letters) 665,L19

Haynes, M. P., \& Giovanelli, R. 1986, ApJ 306, 466

Kent, B. R., Giovanelli, R., Haynes, M. P. et al. 2007, ApJ (Letters) 665, L15

Masters, K. L. 2005, Ph.D. Thesis, Cornell University

Minchin, R., Davies, J., Disney, M., Boyce, P., Garcia, D. et al. 2005a, ApJ (Letters) 622, L21

Minchin, R. F., Davies, J. I., Disney, M. J., Marble, A. R., Impey, C. D., et al. 2005b, astro$\mathrm{ph} / 0508153$

Moore, B., Katz, N., Lake, G., Dressler, A., \& Oemler, A. 1996, Nature 379, 613

Oosterloo, T., \& van Gorkom, J. 2005, A\&\&A 437, L19

Saintonge, A. 2007a, AJ 133, 2087

Saintonge, A. et al. 2007b, $A J$ submitted

Snowden, S. L., et al. 1995, ApJ 454, 643

Solanes, J. M., Sanchis, T., Salvador-Solé, E., Giovanelli, R., \& Haynes, M. P. 2002, AJ 124, 2440

Tonry, J. L., Blakeslee, J. P., Ajhar, E. A., \& Dressler, A. 2000, ApJ 530, 625

Wong, O. I., Ryan-Weber, E. V., Garcia-Appadoo, D. A. et al. 2006, MNRAS , 371, 1855 